\title{
Neuronal communication
}

\author{
We launch a Series on Synaptic Processes, featuring commissioned Reviews and opinion pieces that discuss the \\ expanding molecular understanding of how neural cells communicate.
}

$\mathrm{V}$ isualization of the nervous system in tissue samples under the light microscope became possible around 1873, with Camillo Golgi's silver-stain method revealing a seemingly contiguous entity. In 1888, Santiago Ramón y Cajal introduced the concept that the nervous system is in fact a network composed of individual cells. The term 'synapse', from the Greek word 'synapsis' (meaning 'conjunction'), was used to describe the connection between two neural cells almost a decade later, in 1897, in a physiology textbook by Sir Michael Foster and Charles Scott Sherrington, who followed a suggestion from a classical scholar colleague, Arthur Woollgar Verral.

Synapses are very special connections. A chemical synapse (i.e., one that translates an electrical signal into a chemical one) has a complex architecture and is artistically rendered on this issue's cover. In the axonal terminals of the presynaptic neuron, neurotransmitters are packaged into synaptic vesicles, clustered in the vicinity of the so-called 'active zone', where the release of neurotransmitter occurs. The initial signal is an electric current: an action potential reaches a chemical synapse and causes the opening of voltagesensitive calcium channels, which leads to an increase in the local intracellular calcium concentration; that in turn triggers vesicle fusion and exocytosis, followed by neurotransmitter release. On the other side of the synaptic cleft, the postsynaptic neuron senses the released neurotransmitter via various types of receptors. Ionotropic neurotransmitter receptors are ligandgated ion channels, and metabotropic neurotransmitter receptors, mostly $\mathrm{G}$ protein-coupled receptors, exert their actions through second messengers. The communication through a synapse is tightly regulated by numerous mechanisms that ensure that the message is timely and precise and that the response is appropriate.
For example, monoamine transporters, located on the membrane of the presynaptic neuron, on the periphery of the synapse, mediate reuptake of the excess released neurotransmitter molecules back to the cytoplasm, where they can be repackaged into vesicles.

Researchers now know much more about synapses than did those pioneering neuroscientists who introduced their concept and terminology. Notably, synaptic functions impaired in some diseases can now be modulated. Drugs used to treat depression and anxiety (such as classical tricyclic antidepressants and selective serotonin-reuptake inhibitors) or attentiondeficit/hyperactivity disorder work via a common principle, by increasing the amount of neurotransmitter in the synaptic cleft. Other drugs (e.g., atypical antipsychotic drugs) act directly on the neurotransmitter receptors. Still, there are many unknown factors that can hinder medical progress. For example, identifying pathways that are dysfunctional in a given disorder and those that, even if not directly affected by the disease, are amenable to therapeutic modulation could greatly benefit affected people. Another important application of detailed knowledge of synaptic processes is to diminish or eliminate unwanted side effects associated with the drugs available today.

Recent advances in structural biology, notably in cryo-EM, have provided a wealth of detailed information on membrane proteins, including neurotransmitter receptors and transporters. Light microscopy and sequencing techniques have also offered a window into cellular processes such as localized mRNA translation in neurons. Elucidating the molecular mechanisms behind synaptic functions is essential for reaching a more complete understanding of the synapse and for enabling rational drug development. To highlight the latest progress in this field, we present a special
Series on Synaptic Processes (https://www. nature.com/collections/plqpxptgmh/), featuring commissioned Reviews and opinion articles covering topics from synaptic vesicle fusion to the sensing and recycling of neurotransmitters to localized protein synthesis in neurons. In this issue, we introduce the first three Review articles in this Series.

Bryan Roth writes about a subset of metabotropic neurotransmitter receptors (the G protein-coupled receptors targeted by neuropsychiatric drugs) and explores the links across the structure, function and molecular pharmacology of these molecules ${ }^{1}$. Mary Hongying Cheng and Ivet Bahar review monoamine transporters and discuss how the structural dynamics of these transporters relate to their function and therapeutic modulation ${ }^{2}$. Christine Holt, Kelsey Martin and Erin Schuman examine the experimental evidence for the existence and functional relevance of local protein synthesis in neurons, a process essential for axonal development, synaptogenesis and synaptic plasticity ${ }^{3}$.

This Series will include additional content, to be published in the coming months. In particular, we will explore the mechanisms of synaptic vesicle fusion and neurotransmitter release in a Feature, with contributions from several researchers about the big open questions and challenges in the field. We invite our readers to come back and read these upcoming pieces and hope that all the Series articles will be interesting and inspiring reading.

Published online: 3 July 2019

https://doi.org/10.1038/s41594-019-0265-3

References

1. Roth, B. Nat. Struct. Mol. Biol. https://doi.org/10.1038/s41594019-0252-8 (2019).

2. Cheng, M.H. \& Bahar, I. Nat. Struct. Mol. Biol. https://doi. org/10.1038/s41594-019-0253-7 (2019).

3. Holt, C., Martin, K. \& Schuman, E. Nat. Struct. Mol. Biol. https:// doi.org/10.1038/s41594-019-0263-5 (2019). 